exist, therefore it can't result from repressed childhood abuse, therefore childhood abuse doesn't occur. The backlash False Memory Society has poured phenomenal financial resources into developing a legal counter-culture to contest the work of psychologists and psychiatrists trying to help patients resolve childhood abuse. McHugh's Commentary will only make our job more difficult.

BERKLEy R. POWELL

Clinical Geneticist, Pediatrician

Kapiolani Medical Center for Women \& Children Associate Professor, Pediatrics \& Genetics

John A. Burns School of Medicine

University of Hawaii

Honolulu, Hawaii 96826, USA

McHugh replies - I asserted that psychiatry is a medical discipline vulnerable to faddish errors. My correspondents illustrate how such errors arise.

Dr. Spiegel's letter has many examples. I shall only comment on two. He says "psychiatry did not create dissociation." This is inaccurate. Dissociation is a metaphorical description, coined by a psychiatrist, that often masquerades as an $e x$ planation for certain neurological and psychological artifacts. The artifacts have always existed; the metaphor was new.

The French psychiatrist Pierre Janet first used the term dissociation to describe 'hysterical' symptoms such as artifactual paralyses, sensory losses as well as psychological artifacts such as 'fugues', psychogenic amnesias, MPD etc. He proposed that dissociation was the common element in these artifacts - a disaggregation of the links in consciousness that tied motor, sensory, and affective states together. However, Janet launched a circular argument with his concept.

Thus when a patient presents with several 'personalities', these are 'explained' as dissociative phenomena and the patient said to be suffering a dissociative disorder. However, the evidence for dissociation are the several 'personalities' that dissociation was to explain. One goes round and round this way. The explanatory mechanism - a supposed independent variable - is evoked even though the only indications of it are the symptoms - the dependent variables - to be explained.

The concept of dissociation lacks methodological and conceptual specificity, a problem best appreciated as a mixing of independent and dependent variables. This defect has been recognized by thoughtful psychiatrists right from the start and, when the term dissociation is incáutiously employed, it simply muddles everyone, clinicians and patients alike.

Dr. Spiegel, though, has a penchant for mystifying with metaphor. Thus, he claims that individuals with MPD "suffer not from having more than one personality but from having less than one". This is high-test nonsense. Personality is a loose collective term intended to designate all "which characterizes an individual and determines his unique adaptation to the environment"12. By definition an individual can neither be a fragment of a personality nor several personalities. When a psychiatrist conceptually fractions or multiplies 'personality', he turns a useful psychological concept into Silly Putty.

All three correspondents refer to Terr's opinion that whereas a single trauma is remembered too well by its victim, repeated traumas are "repressed." The essence of this idea is: the more you experience, the less you remember. Quite a claim. One hundred years of continuous investigation into memory has demonstrated just the reverse - repetition of episodes leads to better, not worse recall. As with all dubious clinical ideas, I hold that one should not quote Terr on this matter unless you repeat her work yourself or can point to some prudent investigator who has.

Chefetz obscures the issues with a pseudo-description of psychiatric consultations. No one claims he should stop listening to his patients. But listening is only the clinician's first step. Next, one must evaluate the patients' reports of anxiety, depression, beliefs and other subjective experiences in a critical fashion in order to relieve them - not to elaborate their content or endorse their veracity. Psychiatrists certainly do tell delusional patients that they are mistaken and do not support an 'hysterical' paralysis with crutches. Evaluation - at least on the part of 'serious clinicians' - has always included much more than just listening and acquiescing.

Powell bewilders me with misrepresentations. The False Memory Society (he means the False Memory Syndrome Foundation) does not have "phenomenal financial resources." It has run on a shoestring. Its purpose is not to develop a "counter-culture" but to present scientific facts that will interrupt the ignorant use of psychological and psychiatric notions now fueling a lynch mob.

Also I do not imply that patients with MPD are "liars." I am claiming that because many minds are, as he says "plastic and creative," it is easy for careless therapists to construct artifacts within them of which MPD and false memories are examples.

PAul R. McHugh

Director, Department of Psychiatry

and Behavioral Science

Johns Hopkins School of Medicine

Baltimore, Maryland 21287-7413, USA

1. McHugh, P.R. Witches, multiple personalities, and other psychiatric artifacts. Nature Med. 1, 110-111 (1995).

2. Ameriçan Psychiatric Association: Diagnostic and Statistical Manual of Mental Disorders, Fourth Edition (American Psychiatric Press, Washington, DC, 1994).

3. Kluft, RP. First rank symptoms as diagnostic indicators of multiple personality disorder. Am. J. Psychiat. 144, 293-298 (1987).

4. Boon, S. \& Draijer, N. Multiple personality disorder in The Netherlands: A clinical investigation of 71 patients. Am. J. Psychiat. 150, 489-494 (1993),

5. Terr, L.C. Childhood traumas: An outline and overview. Am. J. Psychiat. 148, 10-20 (1991).

6. Williams, L.M. Recall of childhood trauma: A prospective study of women's memories of child sexual abuse. I. consult. clin. Psychol. 62, 1167-1176 (1994).

7. Spiegel, D. \& Cardeña, E. Disintegrated experience: The dissociative disorders revisited. J. Abnormal Psychol. 100, 366-378 (1991).

8. Koopman, C., Classen, C. \& Spiegel, D. Predictors of posttraumatic stress symptoms among Oakland/Berkeley firestorm survivors. Am. J. Psychiat. 151, 888-894 (1994).

9. Bremner, J.D. et al. Dissociation and posttraumatic stress disorder in Vietnam combat veterans. Am. J. Psychiat. 149, 328-332 (1992).

10. Marmar, C.R. et al. Peritraumatic dissociation and posttraumatic stress in male Vietnam theater veterans. Am. J. Psychiat. 151, 902-907 (1994).

11. Brown, D. Pseudomemories: The standard of science and the standard of care in trauma treatment. Am. J. clin. Hypnosis 37, 1-24(1995).

12. Harch, C.M. \& Schirckel, H.G. Personality Development and Assessment (Ronald, New York, 1950).

\section{The role of clinical research}

To the editor - I applaud your editorial on the increasing endangerment of clinical research and the physician-scientist. In my present position as director of a large research complex with 120 research beds and a mix of over 100 clinical and preclinical researchers all devoted to studying the causes and treatment of psychiatric and neurological disorders, I experience both the pressures and risks of health care cost containment and the glorification of molecular research on a daily basis. 